\title{
Safety risks associated with the lack of integration and interfacing of hospital health information technologies: a qualitative study of hospital electronic prescribing systems in England
}

${ }^{1}$ Centre for Medical informatics, Usher Institute of Population Health Sciences and Informatics, The University of Edinburgh, Edinburgh, UK

Institute for the Study of Science, Technology and Innovation, The University of Edinburgh, Edinburgh, UK

\section{Correspondence to}

Dr Kathrin M Cresswell, Centre for Medical informatics, Usher Institute of Population Health Sciences and Informatics, The University of Edinburgh, Edinburgh EH8 9DX, UK; kathrin.beyer@ed.ac.uk

Received 10 October 2015 Revised 8 March 2016 Accepted 12 March 2016 Published Online First 1 April 2016

\section{SLinked}

- http://dx.doi.org/10.1136/ bmjqs-2017-006661

\section{CrossMark}

To cite: Cresswell KM, Mozaffar $\mathrm{H}$, Lee $\mathrm{L}$,

et al. BMJ Qual Saf 2017;26:530-541.

\begin{abstract}
Background Substantial sums of money are being invested worldwide in health information technology. Realising benefits and mitigating safety risks is however highly dependent on effective integration of information within systems and/or interfacing to allow information exchange across systems. As part of an English programme of research, we explored the social and technical challenges relating to integration and interfacing experienced by early adopter hospitals of standalone and hospital-wide multimodular integrated electronic prescribing (ePrescribing) systems.
\end{abstract}

Methods We collected longitudinal qualitative data from six hospitals, which we conceptualised as case studies. We conducted 173 interviews with users, implementers and software suppliers (at up to three different times), 24 observations of system use and strategic meetings, 17 documents relating to implementation plans, and 2 whole-day expert round-table discussions. Data were thematically analysed initially within and then across cases, drawing on perspectives surrounding information infrastructures.

Results We observed that integration and interfacing problems obstructed effective information transfer in both standalone and multimodular systems, resulting in threats to patient safety emerging from the lack of availability of timely information and duplicate data entry. Interfacing problems were immediately evident in some standalone systems where users had to cope with multiple log-ins, and this did not attenuate over time.
Multimodular systems appeared at first sight to obviate such problems. However, with these systems, there was a perceived lack of data coherence across modules resulting in challenges in presenting a comprehensive overview of the patient record, this possibly resulting from the piecemeal implementation of modules with different functionalities. Although it was possible to access data from some primary care systems, we found poor two-way transfer of data between hospitals and primary care necessitating workarounds, which in turn led to the opportunity for new errors associated with duplicate and manual information transfer. Extending ePrescribing to include modules with other clinically important information needed to support care was still an aspiration in most sites, although some advanced multimodular systems had begun implementing this functionality. Multimodular systems were, however, seen as being difficult to interface with external systems. Conclusions The decision to pursue a strategy of purchasing standalone systems and then interfacing these, or one of buying hospital-wide multimodular systems, is a pivotal one for hospitals in realising the vision of achieving a fully integrated digital record, and this should be predicated on a clear appreciation of the relative trade-offs between these choices. While multimodular systems offered somewhat better usability, standalone systems provided greater flexibility and opportunity for innovation, particularly in relation to interoperability with external systems and in relation to customisability to the needs of different user groups. 


\section{INTRODUCTION}

Health information technology (HIT) is often seen as a solution to current challenges in healthcare including rising costs, growing demands and an ageing population with increasing multiple long-term morbidities. ${ }^{1}$ HIT has been shown to have great potential in this respect through improving processes leading to better outcomes in healthcare safety, quality and efficiency. ${ }^{2}$ A major focus of much current HIT investment involves electronic prescribing (ePrescribing) as it can support decision-making, provide audit trails of medication-related activity and collect data for reuse that can be used to monitor quality and improve processes. $^{4-6}$ ePrescribing has become the focus of efforts for providing information for integrated care that is well coordinated across health and social care settings. This can be achieved by, for example, incorporating additional patient information such as test results to support the quality and safety of prescribing. Furthermore, ePrescribing systems have been shown to reduce medication errors through providing computerised decision support (CDS) at the point of care, to improve legibility by removing the need for handwritten entries that are fraught with error, and to facilitate organisational efficiency. ${ }^{2}$ The implementation of ePrescribing systems is therefore now quite rightly seen by policymakers internationally as a critical step in healthcare reforms and quality improvement initiatives. $^{7-9}$ Implementations are now as a result accelerating across England and many other countries. ${ }^{10}$

In order to achieve the goal of coordinated care, systems need to be connected in order to be able to exchange clinical and other care-related data. ${ }^{11-13}$ One way of achieving this is by interfacing-a strategy that involves linking standalone systems developed separately for different purposes (usually but not always) from different suppliers so that they can exchange information. Standards that guide this information exchange can connect different systems and make them interoperable. Alternatively, adopters may seek to avoid interfacing challenges by procuring hospital-wide multimodular integrated solutions (henceforth described as multimodular systems). These usually take the shape of different system modules (of which ePrescribing may be one) from a single vendor functioning as an integrated whole [eg, an electronic health record (EHR)] with one underlying code and database (although not all multimodular systems have the same underlying code). ${ }^{14-16}$ We have illustrated these concepts in the context of ePrescribing systems (see figure 1). Existing studies reveal an important further distinction between the way that data are processed and presented. ${ }^{17}{ }^{18} \mathrm{~A}$ system may thus be technically integrated (ie, have one underlying code aggregating multiple subsystems that function collectively as one coordinated system), but the end user may not be able to see this integration and benefit from it (eg, they may still need to switch between screens to get all information they need). Similarly, the end user presentation interface may seem integrated (eg, in clinical portals), but the systems may not allow data to be moved seamlessly between applications, and this can create safety risks (eg, relating to moving/transcribing information). ${ }^{19} 20$

For the majority of countries, comprehensive coordination of care and associated quality improvement through HIT is still more of a vision rather than a reality, ${ }^{21}$ but some notable advances have been made within health communities and within organisations such as hospitals, particularly in the USA and parts of Western Europe. ${ }^{22-24}$ For example, in the UK, approaches to implementing ePrescribing systems in hospitals reflect the two strategies surrounding integration and interfacing, with some organisations having implemented multimodular systems integrated with wider hospital information systems, and some implementing standalone ePrescribing solutions. ${ }^{4}$ Keeping in mind that these approaches can also be complementary, studying the implementation of these systems presents an important opportunity to explore
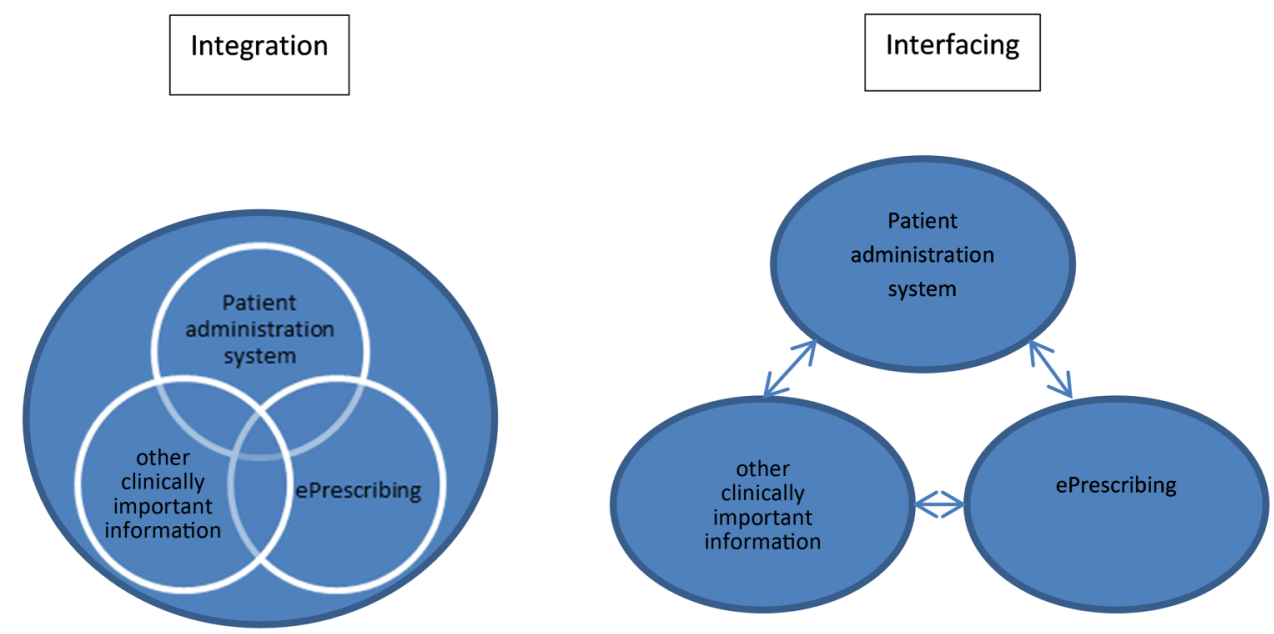
emerging safety risks associated with approaches to integration and interfacing. The problems that arise (for implementers, users and patients), and the strategies to offset these, in relation to design, procurement, implementation and embedding will be crucial for the next generation of health information systems with more sophisticated functionalities in hospitals and their interoperability with wider health and welfare systems. Clinical users in particular need to appreciate underlying performance issues and associated potential threats to safety because these are often not well understood by those faced with the 'front-end' of applications. An appreciation of how the 'back-end' functions is however important to mitigate safety-related risks.

We therefore sought to investigate the two strategies surrounding integration and interfacing in hospitals that have implemented ePrescribing systems, to understand the risks to patient safety arising from the failure of information integration and lack of effective information transfer, and identify potential mitigation approaches. This work is part of an evaluation of ePrescribing systems in English hospitals commissioned by the National Institute for Health Research. ${ }^{4}$

\section{METHODS}

We conducted a longitudinal, qualitative, multisite case study investigating both social and technical challenges relating to interfacing of ePrescribing systems with internal (eg, with EHRs, laboratory data, imaging systems) and external systems (where available, eg, primary care). ${ }^{25}$ Individual hospitals were conceptualised as cases.

\section{Research ethnics committee approval and consent}

This work was reviewed by the London City and East NHS Research Ethics Committee and classified as a service evaluation. We subsequently obtained research ethnics committee approval from The University of Edinburgh and NHS research governance approvals were given by participating hospitals. Individual participants were issued with an information sheet explaining the study and a consent form. Prior to data collection, participants were asked to sign the consent form. In all written material, places/names have been assigned numbers in order to protect anonymity.

\section{Sampling and recruitment}

We purposively sampled a variety of hospital case study sites implementing two different types of ePrescribing systems ${ }^{26}$ : (1) four sites with multimodular ePrescribing systems supplied by one vendor as part of a hospital-wide EHR (ie, providing functionality surrounding ePrescribing, stock control, data analysis and combination with patient notes); and (2) two sites that were coupling ePrescribing solutions with other systems allowing to explore interfacing in they, at the time of recruitment, had either implemented or were planning to implement systems with relevant functionalities. All systems studied were systems that also included discharge prescription and CDS functionality, but greater decision support functionality was available in the multimodular systems.

Within each recruited hospital, we used a combination of purposive and snowball sampling to identify a diverse range of stakeholders. ${ }^{26}$

\section{Data collection}

We used a combination of semistructured interviews, documentary data, observations and expert roundtable discussions (see box 1). ${ }^{27}$

Semistructured interviews were conducted with a diverse range of hospital staff including decisionmakers, implementation teams and information technology staff, clinicians and allied health professionals, and system suppliers (box 2). Topics explored examined backgrounds and experience, perceived challenges surrounding software and hardware integration and interfacing, implications for organisational functioning and work practices, and potential ways to address identified issues (see box 3). We conducted interviews at up to three time points tailored to implementation progress. In organisations that had already implemented a relevant system at the time of recruitment, we conducted two rounds of interviews. This longitudinal design allowed us to investigate expectations, changes over time and varying perceptions as systems were increasingly embedded within organisational functioning. ${ }^{28}$

Targeted non-participant observations complemented interviews (box 2). These were selected based on relevance to ePrescribing-related activity and involved following pharmacists during medicines administration rounds (approximately $3 \mathrm{~h}$ at a time) and attending strategic meetings relevant to ePrescribing strategies (eg, clinical safety groups lasting $1 \mathrm{~h}$ ). This helped us to explore perceived challenges in context and also allowed a detailed insight into the interactions between different stakeholder groups.

Relevant documents were identified by key implementation team contacts at each hospital and included strategic and planning aspects surrounding the implementation. This helped to identify technical features, emerging strategic issues and deviations from original plans.

We also conducted two whole-day expert round-table discussions with 22 participants in order to explore identified challenges surrounding integration and interfacing of systems and implications in more detail (see box 3 for topics explored). Participants included senior clinicians, hospital managers and implementation team leads, representatives from supplier companies, as well as policy and research specialists.

Interviews and round-table discussions were digitally audio-recorded and transcribed verbatim together 
Box 1 Summary of the data set and case study sites

Qualitative data from six case studies-two with standalone electronic prescribing (ePrescribing) systems and four where ePrescribing was part of a multimodular hospital information system:

- 173 interviews with system users, implementers and software suppliers (at up to three different time points)

- 24 observations of system use and strategic meetings (in hospitals A, B and C)

- 17 documents relating to implementation plans

- Two whole-day expert round-table discussions covering four topics with a total of 22 participants from different sectors including clinicians, implementation teams, suppliers, and policy and research specialists.

\section{Case studies}

- Hospital A: urban, acute care, ePrescribing as a standalone application, began implementation in 2010 and had implemented during both data collection periods (time 1, 2 years after implementation; time 2, 4 years after implementation).

- Hospital B: urban, acute care, teaching, ePrescribing as part of a multimodular hospital information system, began implementation in 2009 and had implemented during both data collection periods (time 1, 3 years after implementation; time 2, 5 years after implementation).

- Hospital C: urban, acute care, teaching hospital, ePrescribing as a standalone application, interfaces built to enable interoperability with the wider hospital information systems, implementation started in 2011 (time 1, before system roll-out; time 2, just after the system went live; time 3, 1 year after go-live).

- Hospital D: urban, acute care, teaching, ePrescribing as part of a multimodular hospital information system, implementation started in 2013 (time 1 before system roll-out, time 2 shortly after the system went live).

- Hospital E: urban, acute care, ePrescribing as a standalone application, implementation started in 2011 (time 1, 1 year after start of implementation; time 2, 2 years after the start of implementation; time 3, 3 years after start of implementation; all data were collected pre-implementation).

- Hospital F: urban, acute care, teaching, ePrescribing as part of a multimodular hospital information system, implementation started in 2013 (time 1 before system roll-out, time 2 just after the system went live).

\section{Data analysis}

Data from different sources were coded and organised thematically in NVivo $10 .^{29}$ We began by exploring data within cases (ie, hospitals) and then conducted

\section{Box 2 Interviews and observations}

\section{Interviews}

Site $A$

- Time 1: 23 interviews with users (pharmacists, nurses, doctors) and implementers

- Time 2: 8 interviews with users (pharmacists, nurses, doctors) and implementers

Site B

- Time 1: 20 interviews with users (pharmacists, nurses, doctors) and implementers

- Time 2: 11 interviews with users (pharmacists, nurses, doctors) and implementers

Site $C$

- Time 1: 13 interviews with users (pharmacists, nurses, doctors) and implementers

- Time 2: 18 interviews with users (pharmacists, nurses, doctors) and implementers

- Time 3: 20 interviews with users (pharmacists, nurses, doctors) and implementers

Site $D$

- Time 1: 19 interviews with users (pharmacists, nurses, doctors) and implementers

- Time 2: 7 interviews with users (pharmacists, nurses, doctors) and implementers

- Time 3: 3 interviews with users (pharmacists, nurses, doctors) and implementers

Site $\mathrm{E}$

- Time 1: 24 interviews with users (pharmacists, nurses, doctors) and implementers

- Time 2: 17 interviews with users (pharmacists, nurses, doctors) and implementers

Site $\mathrm{F}$

- Time 1: 15 interviews with users (pharmacists, nurses, doctors) and implementers

- Time 2: 12 interviews with users (pharmacists, nurses, doctors) and implementers

- Time 3: 8 interviews with users (pharmacists, nurses, doctors) and implementers

\section{Observations}

Site A

- Eight observations (12.5 h) of strategic meetings and system use

Site B

- Four observations $(9 \mathrm{~h})$ of strategic meetings and system use

Site C

- Nine observations $(11 \mathrm{~h})$ of strategic meetings and system use

inductive coding approaches permitted us to incorporate emerging themes with existing data and simultaneously allow important new themes to emerge. ${ }^{30}$ In order to achieve this, we drew on a coding framework surrounding the technology lifecycle that we have 
Box 3 Sample interview and round-table discussion topic guide

\section{Interviews}

- Description of background, position, organisational context and system

- Experiences/expectations of system

- Perceived/expected changes in work practices and organisational functioning

- Perceptions on technical interoperability and integration

- Perceptions on end user interoperability and integration

- Perceived benefits/trade-offs of system

Round-table discussions

Part 1: Getting your infrastructure right

- Topic 1: What are the main hard infrastructures that need to be in place?

- Topic 2: What are the main soft infrastructures that need to be in place?

Part 2: Interfacing and integration

- Topic 3: Where is the difference between interfacing and integration?

- Topic 4: What are the main challenges involved in interfacing and integration?

Additional themes were then added as they emerged. Analysis activity also involved actively searching for disconfirming evidence and circumstances that presented disconfirming evidence (negative cases).

In interpreting the findings, we drew on the work surrounding information infrastructures as this can help to conceptualise how different components of an information system function (or not) as a coordinated whole and how this is shaped by context. ${ }^{32-34}$ Information infrastructures are thought to consist of technological and social systems that are linked together to fulfil both local and wider organisational functions. The perspective is therefore helpful in conceptualising both technical and user integration.

\section{RESULTS}

We have identified three main themes (see box 4):

1. lack of effective information transfer and associated safety risks;

2. approaches to improve quality and safety by promoting integration of information;

3. trade-offs between integration and interfacing strategies.

\section{Theme 1: lack of effective information transfer and associated safety risks}

Lack of effective information transfer

In standalone ePrescribing applications, interfaces with other hospital systems had to be created to allow prescribing-related information exchange, but this
Box 4 List of themes and subthemes emerging from this work

Theme 1: lack of effective information transfer and associated safety risks

- Lack of effective information transfer

- Patient safety risks resulting from the lack of effective information transfer

Theme 2: approaches to improve quality and safety by promoting integration of information

- Promoting presentation integration through effective interfacing

- Incorporation of other clinically important information with electronic prescribing

Theme 3: trade-offs between integration and interfacing strategies

- Technical challenges of interoperability in interfacing systems

- Quality of the link between systems

- Monolithic nature of integration route

- Blended approach may be the best way forward

a standalone application, users were faced with multiple log-ins if they wanted to access other systems. For instance, the local Picture Archiving and Communication System required a separate log-in and so did the locally used Patient Administration System (PAS).

So people have to go into one programme to order bloods [blood tests] and review results, they have to go into a different programme to order $\mathrm{x}$-rays and review $\mathrm{x}$-rays, they have to go into a different programme to prescribe. (Hospital A, standalone system, manager, time 2)

Multimodular systems are often perceived to offer seamless transfer of information between different applications and parts of the hospital. However, interfacing with other hospital systems (such as stock control and specialty systems) was still necessary, as specialty systems were used for specific purposes.

[Name of system] is integrated internally, the platform is modular and it sits on a common database... it links to about 20 other downstream systems. (Hospital B, multimodular system, manager, time 1)

A single sign-on that was developed over time helped users to benefit from these interfaces (as multiple long-ins were prevented), but users complained about the time some of these systems took to initialise. In addition, a lack of end user coherence and of seamless navigation between specialty modules and the ePrescribing functionality was viewed as problematic as it necessitated cumbersome switching between screens. This effect was expected to attenuate with the 
but none of the sites included in our study had completed full implementation of all modules.

Hospitals had also begun configuring their systems to interface with primary care applications, which resulted in time savings, as information could be relatively easily accessed, without having to gather it from a variety of different sources. However, although communication between care settings improved over time, in both standalone and multimodular systems, there was still a lack of effective two-way interfacing between hospital and primary care systems.

Patient safety risks resulting from the lack of effective information transfer

We identified a number of safety risks resulting from the lack of effective information transfer in both multimodular and standalone systems. User workloads increased and care activities were slowed down, adversely affecting the timeliness of care provided.

For example, in standalone systems, the lack of effective integration of information between ePrescribing and local PAS and stock control systems resulted in lists of patients not being up-to-date in the ePrescribing system; information entered onto one system was not automatically updated on another system. This lack of availability of up-to-date information created new areas of risk and increased workloads for users (as information had to be transferred manually).

I met the pharmacist in his office and asked him about the stock control system on the way up. This doesn't interface with the ePrescribing system, which means that pharmacy has to print medication lists and then input them manually. (Hospital A, standalone system, observation)

Similarly, the failure to achieve end user coherence and seamless navigation between specialty modules and the ePrescribing functionality in multimodular systems in some instances created new areas of risk and the potential for new errors in entering and accessing data. For example, there was a lack of information transfer from the accident and emergency (A\&E) module in one hospital, resulting in prescribed medications not displaying in the inpatient setting. As a result, users had to switch between the inpatient module and the A\&E module to check whether any medicines had been prescribed. Information was then manually transferred between modules.

...one of the big problems...is the link between [the] A\&E package with the inpatient package and that is an issue because if you've got patients prescribed medications on the [A\&E system] you need to be able to see them on the inpatient record and currently you can't, so currently there's no transfer of that information directly...it is a bit of a safety issue because in order to see what people have had in $[A \& E]$ you have to then look at the [A\&E] discharge summary...I prescribe a drug that someone has already had for example. (Hospital B, multimodular system, consultant, time 2)

This forced users to adopt workarounds to increase speed of system operation.

I actually find it much quicker and easier to keep the PACS system open and access that directly and that's just a simple keyboard shortcut of Alt Shift and you can skip between the two programmes...(Hospital D, multimodular system, consultant, time 2)

Other workarounds involved printing out information from the primary care system (because copying and pasting between systems was not possible), manually re-inputting this into the ePrescribing system, and then printing the information from the ePrescribing system to transfer it back to primary care. It also involved attaching scanned information to patients' records. This opened up possibilities for new errors relating to manual information transfer between systems (especially when the accuracy of transferred information was not double-checked) and was also time consuming for users.

Yeah there are quite a number of things that I think I've found a way round and compensated for one way or the other... there is still a transfer from electronic to paper and then back again to electronic. So there's a lot of work for the pharmacist...(Hospital B, multimodular system, pharmacist, time 1)

\section{Theme 2: approaches to improve quality and safety by promoting integration of information}

Promoting presentation integration through effective interfacing

Some sites with standalone systems had begun to address the issues surrounding presentation integration through effective interfacing, allowing a more coherent end user experience.

[Showing a plan of all the systems and their interface connections] each of these blocks is a separate electronic system for running different parts of the organisation.... (Hospital C, standalone system, manager, time 3)

We also, in some standalone system sites, observed the development of innovative ways to extract and aggregate data from different systems locally. Over time, these resulted in significant benefits for users as they facilitated presentation integration.

We've...linked the database sitting underneath the [ePrescribing] software with our laboratories database so that we can look up a patient's white cell count and what antibiotic they're on, on the same page. (Hospital A, standalone system, manager, time 2)

Incorporation of other clinically important information with ePrescribing Participants across sites viewed linking other clinically important information needed to support care (eg, 
systems as a crucial aspect of future development and system optimisation, and they expected this to result in improved safety and time savings for users from a variety of settings. One way of drawing on this information across system types was to help identify highrisk patients (eg, through searching for those with known problems) and more effectively target actions by pharmacists.

...with ePrescribing I have this vision...you should be able to...know who your high risk patients are and send your more experienced pharmacists to go and see your high risk patients... (Hospital C, standalone system, manager, time 3)

Some hospitals, which had implemented multimodular systems a number of years ago, had begun to link blood glucose measurements and international normalised ratios (INRs) to prescribing. This brought significant benefits and provided the basis for automatic tailoring of medication regimen.

.... lot of the complex prescribing issues aren't just about the medicine, it's about the biochemistry or the results that match that, so with insulin you need to be able to monitor the...blood glucose which in a pure ePrescribing system is difficult. But that's now linked... so the [tests for blood glucose] are immediately available and can be monitored against the insulin...we [also] linked Warfarin dosing with INR's... (Hospital $\mathrm{B}$, multimodular system, manager, time 2)

\section{Theme 3: trade-offs between integration and interfacing strategies}

We identified a number of benefits and trade-offs associated with buying hospital-wide multimodular solutions or multiple standalone solutions, which then need to be knitted together (see table 1). Overall, standalone interfacing solutions were found to present more risks to patient safety relating to information exchange, but were lower in cost and offered greater flexibility and scope for innovation. Multimodular systems were found to facilitate information transfer, but to have disadvantages including high cost and lack of flexibility.

Interfacing was seen to be a problem that needed to be addressed by the hospital implementation team, while integration, particularly in the early stages of implementation, was seen as being a challenge for system developers. In interfacing strategies, there were a number of problems in ensuring reliability, accuracy, consistency and sufficient flexibility in the exchange and retrieval of data, with potentially significant adverse impacts on patient safety. The quality of the link between systems therefore became crucial:

Interoperability is only ever going to be as good as the link between the two systems and how sort of inclusive of information that link is really. (Participant 12, manager, expert round-table discussion 2)

Technical issues were seen as one aspect of the challenges of interfacing as the lack of uniformity and multiple log-ins impacted negatively on the overall usability of systems and the safety of care. Importantly too, the deployment of multiple systems required strong leadership and sound management to ensure that systems remained aligned to the hospital's overall IT deployment strategy.

Although multimodular systems were regarded as helping to address data integration problems, their adopters also expressed some concerns. Although interfacing problems were bypassed within their scope of application, there were still needs to interface with external systems. Moreover, these large multimodular solutions were expensive, rather monolithic and difficult to customise. Meeting the needs resulting from the diversity of local clinical practice was therefore challenging. There were also longer-term dilemmas between upgrading a multimodular package, which might no longer offer the best available solution:

... we bought a [multimodular] system, we've signed a five year contract and at the end of that we're almost certainly going to go with something else. Therefore [ePrescribing system] is going to have to be replaced in

Table 1 Benefits and trade-offs of integration and interfacing approaches

\begin{tabular}{lll}
\hline Benefits & Trade-offs \\
\hline Integration & Consistency & Locked into one supplier \\
& Efficiency & High one-off purchasing cost (as opposed to being able to gradually \\
& Integrated records and improved workflow & purchase complementary functionality) \\
& Ease of use & Limited scope for innovation resulting from new ways to combine software \\
& Costs more predictable/lower maintenance costs & solutions
\end{tabular}

Interfacing Potential to combine with other software to align with local needs

More mature products and better support Best of breed Easier to make changes/smaller system Cost of creating interfaces, potentially lower than multimodular systems

Developed by individual clinical teams, cannot be supported by information technology/isolated expertise Multiple contracts with a number of suppliers Managing multiple supplier relationships Data retrieval

More training required when interacting with several systems Difficulty of getting suppliers to collaborate

Cost and technical challenges in creating interfaces

Lack of standards 
five years with everything else and so, well there are good and bad things with that aren't there? Perhaps one of the advantages about interoperability is that if you can get the systems interfacing OK, then at least you can stick with a decent system, you can go with one that's arguably fit for purpose from the beginning. (Participant 1, pharmacist expert round-table discussion 1)

It was further reported that rolling out a multimodular system and switching from one multimodular system to another was extremely laborious and entailed a high level of risk:

...the change from one system to another is an enormous task. (Participant 13, manager, informatics centre, expert round-table discussion 1)

Despite aiming to provide seamless interoperability of multiple applications, multimodular systems were seen as too deficient in their functionalities. This for some meant that 'integration is not achievable...', in part because suppliers of multimodular systems were unable to keep up with the pace of change in the way care is being delivered.

Overall, participants felt that integration and interfacing strategies had both benefits and risks (table 1). One participant, whose Trust was soon to choose a system, clearly summarised the difficult choice that needed to be made:

We primarily have bespoke systems, best-of-breed, within our organisation we're a best-of-breed scenario but we're still looking at those big solutions like [multimodular system supplier], we recognise that there might be over-riding reasons why you would go for a whole hospital system and that there might be pressure on an organisation to go with that. We also recognise that the standalone systems tend to give you their highest level of functionality per se and possibly the best level of support from around that. But then there are the other disadvantages around interfacing and integration ... there isn't an answer to which one is the best. (Participant 8, pharmacist, expert roundtable discussion 1)

Participants agreed that a compromise would always need to be made when choosing between integration and interfacing strategies. This was especially pronounced in a hospital setting that sought to address the needs of such a varied user base:

You've also got multiple users as well, what I want out of a system is not what, you know, anybody, what the clinicians want out of the system or what the nurses want out of the system so it's always going to be a compromise. (Participant 9, manager, expert roundtable discussion 1)

It emerged that rather than favouring one over the other, participants felt that a combination of described as a blended approach, where bespoke systems could complement multimodular systems:

...even the [multimodular] systems need to be interoperable in a sort of blended approach for both of them. (Participant 4, manager, expert round-table discussion 1)

\section{DISCUSSION}

Integration and interfacing issues in both standalone and multimodular systems can increase user workload, open up possibilities for new errors and have adverse impacts on the timeliness of care provided. Hospitals adopted a number of strategies to address these issues including promoting presentation integration through effective interfacing and incorporating other clinically important information with ePrescribing. Although multimodular systems were valued in terms of usability and in terms of incorporating a wider range of patient information, participants also voiced some concerns that the choice between the strategies of interfacing and integration is an issue of trade-offs that need to be carefully considered before beginning implementation. ${ }^{35}$ Promoting presentation integration of information to the user and maximising clinical benefit through effective interfacing should therefore be a key activity during implementation of both standalone and multimodular systems.

This work, based on a substantial qualitative data set collected from a variety of sources, has provided a range of perspectives and an appreciation of how interfacing and presentation integration issues can persist after implementation. ${ }^{28}{ }^{36}$ In doing so, it offers unique insights into the issues and potential surrounding integration and interfacing in different types of ePrescribing systems being implemented in the UK. However, as with all qualitative research it is based on perceptions, perspectives and experiences. ${ }^{37}$ Therefore, insights obtained based on participant experiences complicates tracing of impacts on delivery of care and underlying technical issues. In this respect, it is important to keep in mind that there tend to be reasons underlying strategic decisions, which may include technical constraints. These need to be understood in order to attain a comprehensive picture of the implementation, adoption and optimisation landscape. Other limitations include the fact that this work is based on a limited number of settings, which may not be representative of the wider range of other UK hospitals. Although we did obtain some insights into developments over time, it is important to keep in mind that we did not follow-up developments over long time periods, which potentially could have provided insights into ways that sites addressed these emergent interfacing and integration issues. This is a key activity we will aim to pursue in future work.

We have obtained important insights relating to the 
solutions and configurations of linked standalone elements) and multimodular ePrescribing systems (table 1). While multimodular systems offer the potential for an improved user experience and facilitate the availability of information in one place (even if this place is modular), they also limit the potential for customisation and innovation, and mean that hospitals are locked in contracts with a single supplier. Multimodular ePrescribing systems may be seen as a 'base' as they cover many different functionalities, of which ePrescribing is just one. ${ }^{32}$ New interfaced systems can be added, but these need to fit in with what is already there-in some ways, multimodular systems can therefore limit improvements in design and also usability. Interfaced systems, on the other hand, do offer increased flexibility, but present issues in relation to incorporation of information and user experience.

However, participants also highlighted that high levels of integration from the perspective of one set of users may not meet the needs of other sets of users. This is because in healthcare different groups of users have varying needs, which need to be considered and, if possible, catered for. ${ }^{38} 39$ Implementers' and policymakers' focus on integration, with its emphasis on standardisation of practices, may in fact hinder achieving these information needs. ${ }^{40}$ The highest desirable level of integration should therefore consist of an overview of information for all user groups, with various professions having to go into different modules of the system for detailed information, necessitating switching between screens (as we observed in multimodular systems). Such a model also exists in enterprise systems, ${ }^{41-43}$ where users have to navigate through a variety of screens to cater for the needs of different organisational players, thereby compromising usability for individual user groups. ${ }^{44} 45$ Approaches to address these challenges in enterprise systems have included changing user interfaces through flexible screens, optimising screen flows and implementing multiple ways to reach the same information based on responsibility/profession. ${ }^{4-46}$

Both integration (ie, procuring hospital-wide systems from a single supplier) and interfacing (ie, knitting together various standalone packages) strategies have advantages and trade-offs. To build on this finding, we suggest exploring whether a combination of integration and interfacing approaches is likely to be feasible and how this may be conceptualised in order to maximise benefits. ${ }^{47} 48$ This should involve organisational strategies surrounding choice and integration of basic organisational systems (such as EHRs) and effectively interfacing these with specialty systems. In doing so, Trusts should consider the merits and disadvantages of both approaches and then reflect on both their internal vision, needs and capabilities, and the relative benefits and trade-offs before deciding on the course of action. If hospitals only have limited internal capacity to interface with other software systems or to outsource this work, a multimodular

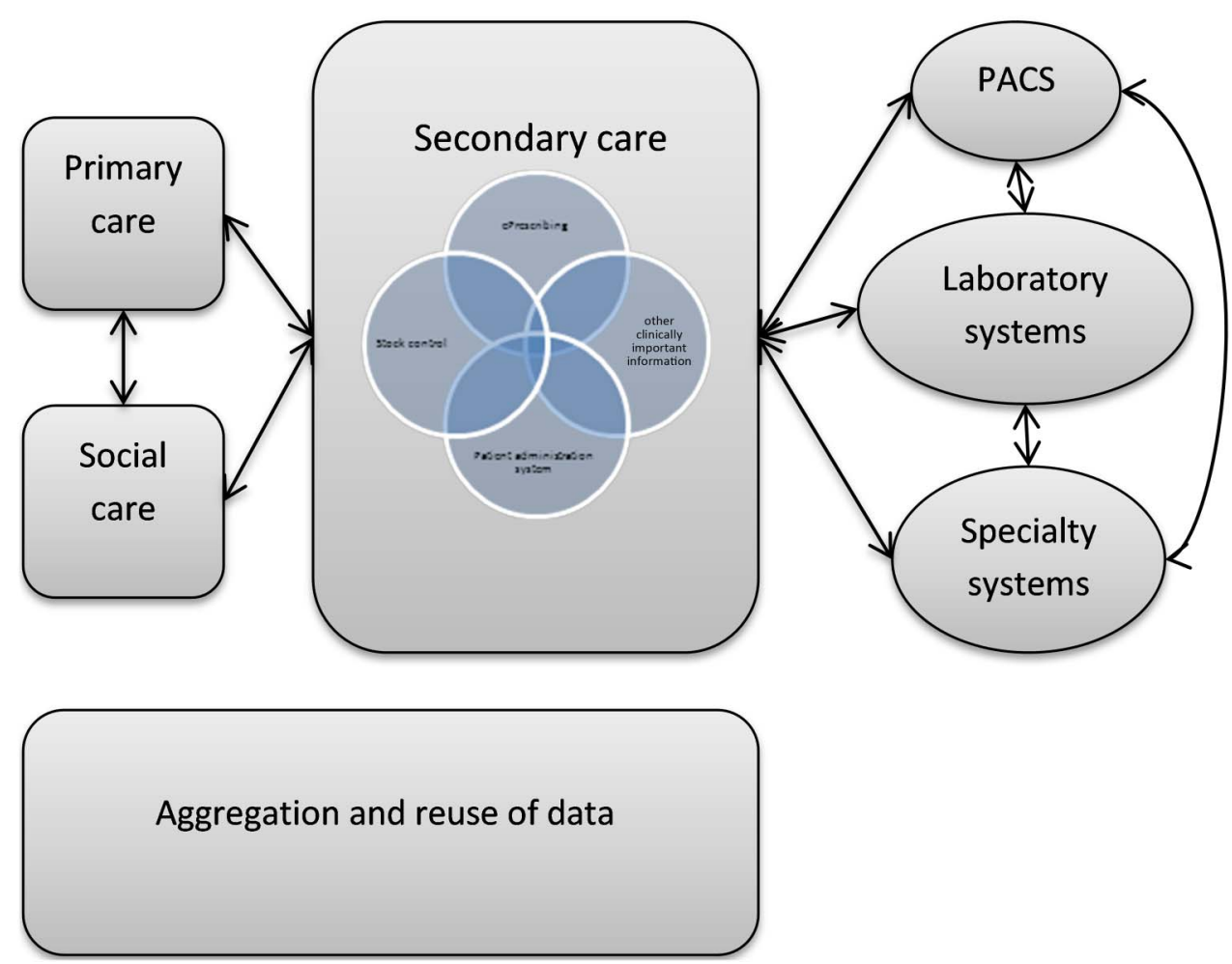

Figure 2 A visualisation of an idealised multimodular electronic prescribing (ePrescribing) system interfacing with other care settings 
hospital-wide system is likely to be the safer option to pursue. Our work also highlights the fact that in choosing systems, decision-makers have to trade off a range of considerations, of which patient safety is only one. More research into the advantages and disadvantages of integration and interfacing strategies in different contexts and how best to mix these approaches is clearly needed to build on insights emerging from this work. This will also need to involve more in-depth work on developing theoretical models surrounding these constructs. ${ }^{49}$

There is currently a lack of national technical and process standards for joining up systems, and this may limit opportunities to select bespoke systems suited to individual specialties. More flexible interfacing (eg, between ePrescribing and specialty systems) should also be considered by system developers if they want their systems to be used more widely. Addressing this issue may further help meet users' and patients' needs, ${ }^{50}$ facilitating more seamless switching between functionalities to promote end user coherence. A potential architecture of how a multimodular local hospital ePrescribing system may interface with other care settings is given in figure 2 . In order to achieve this, more immediate system optimisation through integration and interfacing should focus on enhancing the user experience through amalgamation of information (particularly in the early stages of implementation when parallel systems are the norm); incorporation of other clinically important information and ePrescribing as soon as possible as this is expected to bring the biggest benefits; developing two-way data exchange of minimum data sets between primary and secondary care interfaces; and exploring ways to aggregate data from different systems/sectors to enhance innovative secondary uses. ${ }^{51}$

Other fruitful areas for further work include investigating which approach is best suited for larger groupings of hospitals and how appropriate application programme interfaces can be developed to promote local innovation.

\section{CONCLUSIONS}

This work has explored the integration and interfacing strategies and problems encountered when implementing ePrescribing systems. Both strategies require attention to be paid to interfacing and we also observed integration problems with multimodular solutions. The pursuit of information integration will require attention to be paid to both integration and interfacing challenges and associated activities with the aim to create integrated information systems/infrastructures. Irrespective of the approach, a greater focus on user needs, attention to usability, and wider social and technical considerations is important in order to move towards seamless presentation of information to end users in a way that can be readily
We have provided guidance for policymakers in defining potential future strategies for investment into the development of new health and care services and national health infrastructures. These efforts should be characterised by future work devising interoperability standards that allow innovative and safe ways to connect ePrescribing systems with wider organisational processes and information held in related systems. Our work can also help to improve understanding of how best to build and implement systems with integration and interfacing in mind, thereby maximising potential benefits from the start.

Acknowledgements We gratefully acknowledge the input from our Independent Programme Steering Committee, which is chaired by Prof Denis Protti and has Prof Sir Munir Pirmohamed, Prof Bryony Dean Franklin, Ms Eva Leach, Ms Rosemary Humphreys, and Ms Ailsa Donnelly as members. We also gratefully acknowledge the input of Rosemary Porteous (RP), who transcribed the discussions. Members of the Programme Team are: Dr Ann Robertson, Prof Jill Schofield, Dr Jamie Coleman, Ms Ann Slee, Prof David Bates, Dr Zoe Morrison, Mr Alan Girling, Mr Antony Chuter, Dr Laurence Blake, Prof Anthony Avery, Prof Richard Lilford, Dr Sarah Slight, Dr Behnaz Schofield, Ms Sonal Shah, Ms Ndeshi Salema, Mr Sam Watson, and Dr Lucy McCloughan.

Contributors AS and RW conceived this work. LL, KMC and HM collected data for this study. KMC led on data analysis and drafting of the manuscript. All authors have commented on various versions of this manuscript and inputted into the analysis.

Funding National Institute for Health Research (RP-PG-1209-10099).

Competing interests None declared.

Patient consent Not applicable.

Ethics approval The University of Edinburgh.

Provenance and peer review Not commissioned; externally peer reviewed.

\section{REFERENCES}

1 Suzman R, Beard JR, Boerma T, et al. Health in an ageing world-what do we know? Lancet 2015;385:484-6.

2 Black AD, Car J, Pagliari C, et al. The impact of eHealth on the quality and safety of health care: a systematic overview. PLoS Med 2011;8:e1000387.

3 Buntin MB, Burke MF, Hoaglin MC, et al. The benefits of health information technology: a review of the recent literature shows predominantly positive results. Health Aff (Millwood) 2011;30:464-71.

4 Cresswell KM, Bates DW, Williams R, et al. Evaluation of medium-term consequences of implementing commercial computerized physician order entry and clinical decision support prescribing systems in two 'early adopter' hospitals. J Am Med Inform Assoc 2014;21:e194-202.

5 Overhage JM, Gandhi TK, Hope C, et al. Ambulatory computerized prescribing and preventable adverse drug events. J Patient Saf 2015.

6 Cresswell K, Majeed A, Bates DW, et al. Computerised decision support systems for healthcare professionals: an interpretative review. Inform Prim Care 2012;20:115-28.

7 NHS England. The Integrated Digital Care Fund. Achieving integrated health and care records. http://www.england.nhs.uk/ wp-content/uploads/2014/05/idcr.pdf (last accessed 08 Jul 
8 Blumenthal D, Tavenner M. The "Meaningful Use" regulation for electronic health records. N Engl J Med 2010;363:501-4.

9 Connor M, Day GE, Meston D. Successful linking of patient records between hospital services and general practice to facilitate integrated care in a hospital and health service in south-east Queensland. Aust Health Rev 2015.

10 Ahmed Z, McLeod MC, Barber N, et al. The use and functionality of electronic prescribing systems in English acute NHS trusts: a cross-sectional survey. PloS ONE 2013;8: e80378.

11 Kodner DL, Spreeuwenberg C. Integrated care: meaning, logic, applications, and implications-a discussion paper. Int J Integr Care 2002;2:e12.

12 World Health Organization. Integrated health services-what and why? http://www.who.int/healthsystems/technical_brief_ final.pdf (last accessed 08 Jul 2015).

13 Fleck J. The Development of information integration: beyond CIM? Edinburgh PICT Working Paper, No 9, Research Centre for Social Sciences, Edinburgh University, 1988.

14 Mordecai Y, Dori D. A model-based framework for architecting system-of-systems interoperability, interconnectivity, interfacing, integration, and interaction. INCOSE Int Symp 2013;23:1234-55.

15 Wainwright $\mathrm{D}$, Waring T. The information management and technology strategy of the UK National Health Service-Determining progress in the NHS acute hospital sector. Int J Public Sector Manag 2000;13:241-59.

16 NHS England. Safer hospitals safer wards: achieving an integrated digital care record. https://www.england.nhs.uk/ wp-content/uploads/2013/07/safer-hosp-safer-wards.pdf (last accessed 08 Mar 2016).

17 Ellingsen G, Monteiro E. The organizing vision of integrated health information systems. Health Informatics $J$ 2008;14:223-36.

18 Carter J. Electronic health records: a guide for clinicians and administrators. Philadelphia, PA: American College of Physicians, 2001.

19 Røstad L, Nytrø Ø, Tøndel IA. Access control and integration of health care systems: an experience report and future challenges. In Availability, Reliability and Security. ARES 2007. The Second International Conference on. IEEE, 2007:871-8.

20 Berger RG, Baba J. The realities of implementation of Clinical Context Object Workgroup (CCOW) standards for integration of vendor disparate clinical software in a large medical center. Int J Med Inform 2009;78:386-90.

21 King's Fund. Making integrated care happen at scale and pace. http://www.kingsfund.org.uk/sites/files/kf/field/field_ publication_file/making-integrated-care-happen-kingsfundmar13.pdf (last accessed 08 Jul 2015).

22 Jha AK, DesRoches CM, Kralovec PD, et al. A progress report on electronic health records in US hospitals. Health Aff (Millwood) 2010;29:1951-7.

23 Chen C, Garrido T, Chock D, et al. The Kaiser Permanente Electronic Health Record: transforming and streamlining modalities of care. Health Aff (Millwood) 2009;28:323-33.

24 Sheikh A, Cornford T, Barber N, et al. Implementation and adoption of nationwide electronic health records in secondary care in England: final qualitative results from prospective national evaluation in "early adopter" hospitals. BMJ 2011;343:d6054.

25 Crowe C, Cresswell K, Robertson R, et al. The case study approach. BMC Res Methodol 2011;11:100.
26 Coyne IT. Sampling in qualitative research. Purposeful and theoretical sampling; merging or clear boundaries? J Adv Nurs 1997;26:623-30.

27 Guion LA, Diehl DC, McDonald D. Triangulation: establishing the validity of qualitative studies. University of Florida: IFAS Extension, 2011.

28 Saldaña J. Longitudinal qualitative research: analyzing change through time. Altamira: Rowman, 2003.

29 QSR International. NVivo 10 for Windows. http://www. qsrinternational.com/products_nvivo.aspx (last accessed $08 \mathrm{Jul}$ 2015).

30 Denzin NK, Lincoln YS. The SAGE handbook of qualitative research. Thousand Oaks: SAGE, 2005.

31 Cresswell K, Coleman J, Slee A, et al. A toolkit to support the implementation of electronic prescribing systems into UK hospitals: preliminary recommendations. $J$ R Soc Med 2014;107:8-13.

32 Hanseth O. From systems and tools to networks and infrastructures-From design to cultivation. Towards a theory of ICT solutions and its design methodology implications. http://heim.ifi.uio.no/ oleha/Publications/ib_ISR_3rd_resubm2. html (last accessed 08 Jul 2015).

33 Pollock N, Williams R. E-infrastructures: How do we know and understand them? Strategic ethnography and the biography of artefacts. Comput Support Cooperat Work 2010;19:521-56.

34 Aanestad M, Blegind Jensen T. Building nation-wide information infrastructures in healthcare through modular implementation strategies. J Strateg Info Systems 2011;20:161-76.

35 Koch C. Production management systems-bricks or clay in the hands of the social. Technol Anal Strateg Manage 1997;12:119-38.

36 Guest G, Bunce A, Johnson L. How many interviews are enough? An experiment with data saturation and variability. Field Methods 2006;18:59-82.

37 Patton MQ. Qualitative research. John Wiley \& Sons, Ltd, 2005.

38 Berg M. Implementing information systems in health care organizations: myths and challenges. Int J Med Inform 2001;64:143-56.

39 Berg M. Patient care information systems and health care work: a sociotechnical approach. Int J Med Inform 1999;55:87-101.

40 Ellingsen G, Monteiro E. Seamless integration: standardisation across multiple local settings. Comput Support Cooperat Work 2007;15:443-66.

41 Sanner MF. Python: a programming language for software integration and development. J Mol Graph Model 1999;17:57-61.

42 Woyak SA, Myklebust A. Functionality and data integration of software modules through dynamic integration. J Engineering Design 1998;9:137-58.

43 Barrett DJ, Clarke LA, Tarr PL, et al. A framework for event-based software integration. ACM Trans Software Eng Method 1996;5:378-421.

44 Soh C, Sia SK, Boh WF, et al. Misalignments in ERP implementation: a dialectic perspective. Int J Hum-Comput Interact 2003;16:81-100.

45 Sia SK, Tang M, Soh C, et al. Enterprise Resource Planning (ERP) systems as a technology of power: empowerment or panoptic control? ACM Sigmis Database 2002;33: 23-37. 
46 Calisira F, Calisirb F. The relation of interface usability characteristics, perceived usefulness, and perceived ease of use to end-user satisfaction with enterprise resource planning (ERP) systems. Comput Hum Behav 2004;20:505-15.

47 Humphries R. Integrated health and social care in EnglandProgress and prospects. Health Policy 2015;119:856-9.

48 Hutchison KF. An exploration of the integration of health and social care within Scotland: senior stakeholders views of the key enablers and barriers. J Integr Care 2015;23:129-142.
49 Waring T, Wainwright D. Interpreting integration with respect to information systems in organizations-image, theory and reality. J Info Technol 2000;15:131-47.

50 Lee L, Williams R, Sheikh A. What Does ePrescribing mean for patients? A case study of the perspectives of hospital renal patients. J Innov Health Inform 2015;22:391-8.

51 Atherton IM, Lynch E, Williams AJ, et al. Barriers and solutions to linking and using health and social care data in Scotland. Br J Soc Work 2015;45:1614-22. 